\title{
Chronic Subdural Haematoma in a Case of Hyperthyroidism Presenting with Papilledema
}

\author{
Somya Dulani*, Rajesh Dulani, Seema Lele, Sachin Diagavane, \\ Sandeep Anjankar, Netra Jaiswal, Prem S. Subramaniam, Rakesh Juneja \\ Ophthalmology JNMC DattaMeghe, Institute of Medical Sciences, Wardha, India \\ Email: *somya1010@rediffmail.com, rajeshkdulani@gmail.com,drseemalele@gmail.com,drsachin391977@gmail.com, \\ drsdanjankar@yahoo.co.in,netra_jaiswal@yahoo.com, psubram1@jhmi.edu,dr_rakeshjuneja555@yahoo.com
}

Received August 28, 2012; revised October 13, 2012; accepted October 25, 2012

\begin{abstract}
Subdural hematomas are often life-threatening when acute but chronic subdural hematomas, however, have better prognosis if properly managed. Chronic subdural hematomas are common in the elderly due to shrinkage of brain tissue, but in young patient mostly associated with head injury. It is seen also in young having various coagulopathies associated with blood disorders or drug-induced, but it is very rare. Propylthiouracil (PTU) is an oral medication that is used in treatment of hyperthyroidism approved by FDA in July 1947. This medication may rarely cause very serious blood disorders (such as a low number of red cells, white cells, and platelets), especially during the first few months of treatment. We are reporting a rare case of PTU-induced thrombocytopenia leading to chronic subdural haematoma, which presented with established papilledema and signs of raised ICP in a hyperthyroid female and she responded well to surgical management.
\end{abstract}

Keywords: Propylthiouracil (PTU); Thrombocytopenia; Papilledema

\section{Introduction}

A subdural hematoma, a form of traumatic brain injury in which, blood gathers within the outermost meningeal layer, between the dura mater, which adheres to the skull, and the arachnoid mater, which envelops the brain. Usually resulting from tears in bridging veins which cross the subdural space, subdural hemorrhages may cause an increase in intracranial pressure (ICP), which can cause compression of and damage to delicate brain tissue. Chronic subdural bleeds develop over a period of days to weeks, often after minor head trauma, though such a cause is not identifiable in $50 \%$ of patients [1]. Head injury is the most common cause of this lesion, but several predisposing factors such as coagulopathy, alcoholism, cerebrospinal fluid shunt procedures, vascular malformations, seizure disorders, and metastatic tumours must also be considered [2]. Hyperthyroidism, a hypermetabolic clinical syndrome which occurs, when there are elevated serum levels of T3 and/or T4. As there is too much thyroid hormone, every function of the body tends to speed up with symptoms like nervousness, irritability, increased perspiration, heart racing, hand tremors, anxiety, difficulty sleeping, thinning of the skin, fine brittle hair, and muscular weakness.

${ }^{*}$ Corresponding author.
Thyrostatics (antithyroid drugs) are drugs that inhibit the production of thyroid hormones, such as carbimazole (used in UK) and methimazole (used in US), and propylthiouracil. Propylthiouracil (PTU) also works outside the thyroid gland, preventing conversion of (mostly inactive) $\mathrm{T} 4$ to the active form T3. It decreases production of thyroid hormone and also interferes with the conversion of $\mathrm{T} 4$ to $\mathrm{T} 3$, and, since $\mathrm{T} 3$ is more potent than $\mathrm{T} 4$, this also reduces the activity of thyroid hormones. In most cases, adverse effects are minor and transient. The most dangerous effect is agranulocytosis, which occurs in 0.1 to $0.5 \%$ of patients. Other major adverse effects aplastic anaemia, thrombocytopenia, lupus erythematosus-like syndrome, vasculitis are exceedingly rare [3].

\section{Case Report}

A forty five year hyperthyroid female (Figure 1) presented in eye clinic with a complaint of severe headache in fronto-parietal region which was radiating in the neck. Along with throbbing headache she also had nausea and vomiting since one month. Previously one year back, she presented with similar complaints of raised intracranial pressure. At that time she was onantithyroids drugs for hyperthyroidism since three years and but inadequate treatment as her T3-517 ng/dl, T4 > 30 ng/dl and TSH-13 $\mathrm{ul} / \mathrm{mL}$. She was advised to take adequate treatment and 


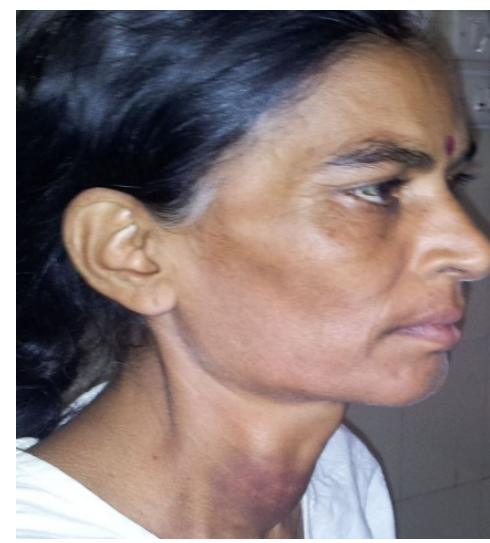

Figure 1. Clinical photograph of patient with mild swelling in neck.

her symptoms responded well to the treatment. But this time, patient was on regular antithyroid treatment and her thyroid profile was normal, so she was evaluated further. On ocular examination her visual acuity was 20/30 in both eyes with Marcus - Gunn pupillary reaction in both eyes. Fundus examination revealed well established papilledema in both eyes with disc swelling more than 5 disc diopters in both eyes (Figure 2).

She was advised MRI of brain, to rule out space occupying lesion which revealed, subacute extra-axial (subdural) hemorrage in right frontoparieto and occipital region in different stages causing midline shift to left side by $4 \mathrm{~mm}$ with cerebral oedema (Figures 3 and 4).

She had no history of head injury in past years. Her haemoglobin was $9.3 \mathrm{gm} \%$ and platelet count was very low that is $40,000 /$ cu.mm. Systemic examination was done but other signs of thrombocytopenia were not present except excessive menstrual bleeding. Her drug history was taken, she was on PTU, a drug used as antithyroids since 3 months. She was advised to stop PTU drug and carbimazole was started. She was operated for subdural haematoma by a neurosurgeon after Fresh Frozen Plasma (FFP) transfusion to increase platelet count. Her post operative period was uneventful and within 2 days her papilledema reduced and on 5 th day she had near to normal optic disc (Figure 5).

\section{Discussion}

One of the rare side effect of PTU is, decrease in blood platelets (thrombocytopenia). Since platelets are important for the clotting of blood, thrombocytopenia may lead to excessive bleeding [4]. A study of Subduralhaematoma among people who have hyperthyroidism, regardless of type of antithyroid drug taken was done [5]. On 2 July 2012, 1604 people who have hyperthyroidism are studied. Among them, $2(0.12 \%)$ have Subdural Haematoma, and both were female, though the cause was not discussed. In our case probable cause is PTU-induced.

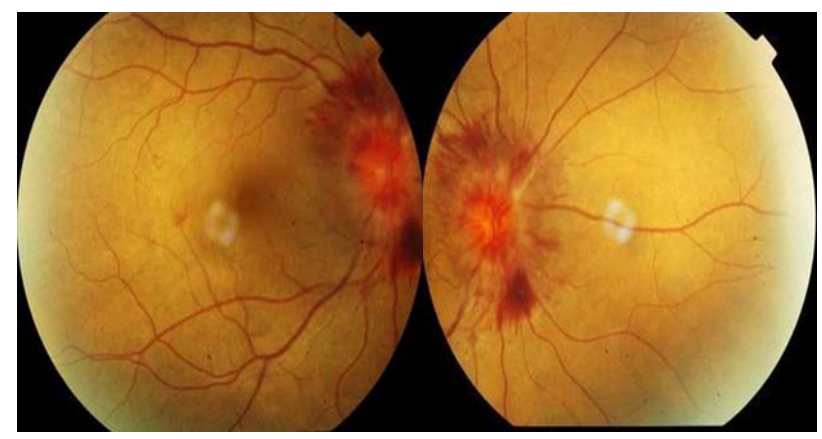

Figure 2. Fundus photograph showing established papilledema in both right and left eye before treatment.

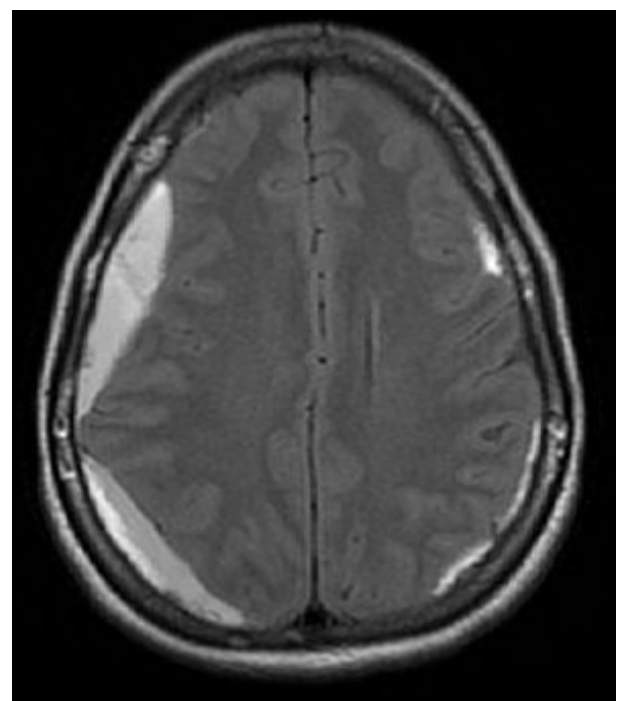

Figure 3. T-1 weighted Axial section MRI shows subacute extra-axial (subdural) hemorrhage in right frontoparieto and occipital region causing midline shift to left side by 4 mm with cerebral oedema.

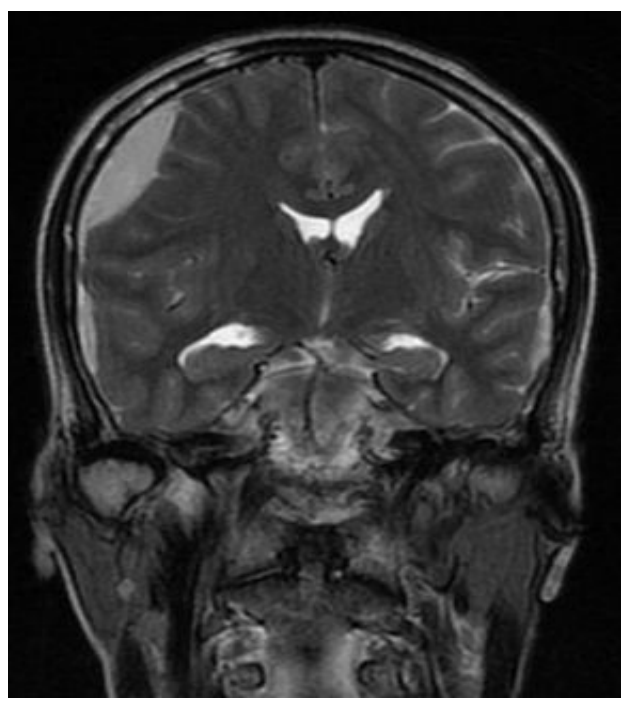

Figure 4. T-2 weighted Coronal section MRI shows subdural hemorrhage in parietal and occipital region causing midline shift to left side. 


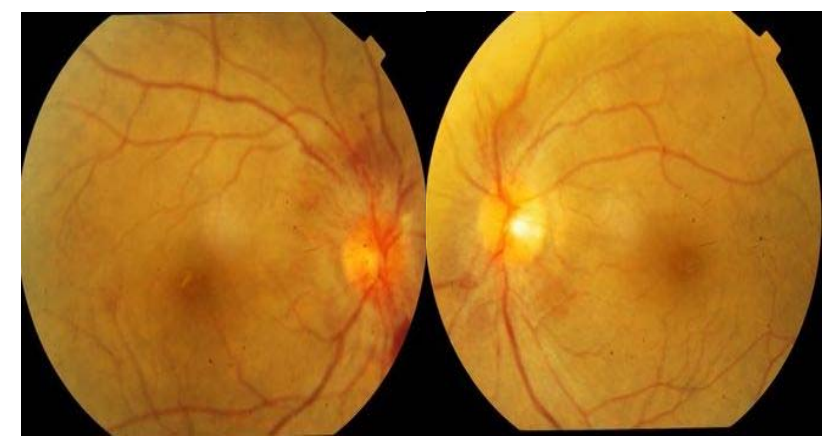

Figure 5. Fundus photograph showing resolving papilledema in both right and left eye after surgical management of subdural haematoma.

Yaka [6] and Herwig [7] presented a case of hyperthyroidism which was associated with symptoms of raised intracranial pressure which responded to antithyroids drugs because it was due to hyperactivity of thyroid hormone, similarly in our case first presentation that is one year before was due to deranged thyroid profile but second time it was due to chronic subdural haematoma.

Pseudotumorcerebri (PTC) is an entity characterized by elevated intracranial pressure with normal cerebrospinal fluid (CSF) and no structural abnormalities detected on brain MRI scans. The neurological symptoms and signs can be totally attributed to intracranial hypertension, and papilledema being the hallmark of PTC and its common cause is hyperthyroidism [8]. Thyroid testing may be useful for differential diagnosis of chronic headache, and indicates that headache could be caused by hyperthyroidism [9].

Chronic subdural hematoma is an important reversible cause of dementia and disability in the elderly. A sufficiently high level of clinical suspicion and prompt radiographic evaluation may allow for timely treatment to avoid poor outcomes. Thankfully, the routine use of computed tomographic scanning in most emergency facilities has made the diagnosis of these lesions common place [10]. Though there are cases of hyperthyroid females reported in literature associated with optic disc swelling, but drug-induce chronic subdural haematoma leading to papilledema in these patient is not reported till date.

\section{REFERENCES}

[1] A. Downie, "Tutorial: CT in Head Trauma," 2007.

[2] A. Prystupa, E. Kurys-Denis, T. Łopatyński, J. Baraniak and W. Krupski, "Case Report: Chronic Subdural Haematoma in a Patient with Arterial Hypertension and Alzheimer's Disease," Journal of Pre-Clinical and Clinical Research, Vol. 3, No. 2, 2009, pp. 127-129.

[3] L. Bartalena, F. Bogazzi and E. Martino, "Adverse Effects of Thyroid Hormone Preparations and Antithyroid Drugs," Adverse Effects of Thyroid Hormone Preparations and Antithyr, Vol. 15, No. 1, 1999, pp. 53-63. doi:10.2165/00002018-199615010-00004

[4] Medicine Net.com, "Newsletter Medicationspropylthiouracil Drug Monograph,” 2012. http://www.medicinenet.com

[5] eHealth Me, "Real World Drug Outcomes. Could Hyperthyroidism Causes Subdural Haematoma," 2012. http://www.ehealthme.com

[6] E. Yaka and R. Çakmur, "Increased Intracranial Pressure Due to Hyperthyroidism," Cephalalgia, Vol. 30, No. 7, 2010, pp. 878-880.

[7] U. Herwig and M. Sturzenegger, "Hyperthyroidism Mimicking Increased Intracranial Pressure," Headache: The Journal of Head and Face Pain, Vol. 39, No. 3, 1999, pp. 228-230.

[8] E. Coutinho, A. M. Silva, C. Freitas1 and E. Santos, "Graves' Disease Presenting as Pseudotumorcerebri: A Case Report," Journal of Medical Case Reports, Vol. 5, 2011, p. 68. doi:10.1186/1752-1947-5-68

[9] U. Herwig and M. Sturzenegger, "Brief Communication: Thyroid Function in Patients with Chronic Headache," International Journal of Neuroscience, Vol. 57, No. 3-4, 1991, pp. 263-267.

[10] J. C. T. Chen and M. L. Levy, "Causes, Epidemiology and Risk Factors of Chronic Subdural Hematoma," Neurosurgery Clinics of North America, Vol. 11, No. 3, 2000, pp. 399-406. 